\title{
Paraneoplastic neurological syndrome presenting as difficulty in mouth opening in a patient with small cell lung cancer
}

\author{
Hirohide Kutsuma $\cdot$ Kazunari Yamana $\cdot$ Yoshiaki Nagai · Shintaro Mikami • \\ Hiroyuki Kyoyama - Yuriko Saito · Ryu Ono • Toru Noguchi - Gaku Moriyama • \\ Kazuo Yamamoto $\cdot$ Kyoichi Nomura $\cdot$ Kazutsugu Uematsu
}

Received: 4 November 2011/ Accepted: 5 March 2012/Published online: 30 March 2012

(C) The Japan Society of Clinical Oncology 2012

\begin{abstract}
A 60-year-old man was admitted to our hospital experiencing difficulty in opening his mouth, and diplopia. Chest computed tomography showed a mass on the right lung. Transbronchial biopsy and scrutiny of the whole body resulted in the diagnosis of small cell lung cancer, cT2N2M1a stage IV. With chemotherapy including carboplatin and etoposide, the primary mass of the right lung shrank, and the patient no longer had diplopia or trouble opening his mouth. After the first-line chemotherapy, itching, ptosis, and dysphagia appeared, accompanied by tumor relapse. These symptoms improved with chemotherapy for lung cancer. After four lines of chemotherapy, the lung cancer was not controlled. The patient died 19 months after the first chemotherapy had started. Difficulty in opening the mouth is a rare symptom for lung cancer patients, but chemotherapy for lung cancer clearly improved this symptom. Therefore, we diagnosed this rare symptom as paraneoplastic neurological syndrome.
\end{abstract}

Keywords Paraneoplastic neurological syndrome . Difficulty in opening the mouth · Small cell lung cancer

H. Kutsuma · K. Yamana · Y. Nagai · S. Mikami ·

H. Kyoyama · Y. Saito · R. Ono · T. Noguchi · G. Moriyama ·

K. Yamamoto $\cdot$ K. Uematsu $(\bowtie)$

Department of Pulmonary Medicine, Saitama Medical Center,

Saitama Medical University, 1981 Kamoda, Kawagoe, Saitama

350-8550, Japan

e-mail: kuematsu@saitama-med.ac.jp

K. Nomura

Department of Neurology, Saitama Medical Center, Saitama

Medical University, Kawagoe, Saitama 350-8550, Japan

\section{Introduction}

Paraneoplastic neurological syndrome shows various symptoms and often accompanies small cell lung cancers. We used chemotherapy to treat small cell lung cancer in a patient who had difficulty opening his mouth. This symptom disappeared as the lung cancer lesions shrank. This phenomenon is very rare, but we diagnosed this symptom as paraneoplastic neurological syndrome by applying a set of diagnostic criteria recommended by the Paraneoplastic Neurological Syndrome Euronetwork [1].

\section{Case report}

A 60-year-old man had general fatigue and low-grade fever for 2 weeks, and so he sought treatment at a hospital. Blood chemistry and chest radiogram were assessed but there were no abnormal findings. Two weeks after those tests had been performed, he began to have difficulty opening his mouth, and he lost the ability to eat. He then presented to a hospital of otorhinolaryngology, and left recurrent laryngeal nerve paralysis was found. He was immediately admitted to the emergency room of our hospital. On admission, he could not open his mouth more than $2 \mathrm{~cm}$, but his mouth could be forced to open $3 \mathrm{~cm}$. He could not push his tongue out. His oral cavity contained no dental abscess or extraordinary findings. His consciousness level was clear and he was able to converse. His blood pressure was $157 / 83 \mathrm{mmHg}$ and his heart rate was 80 beats/min. His pupils were symmetrical and his light reflex was prompt bilaterally. Diplopia was found when he focused to the right. Neither nystagmus nor ptosis was found. Coordination was normal. No motor paralysis of the muscles of his limbs was found, nor was there any 


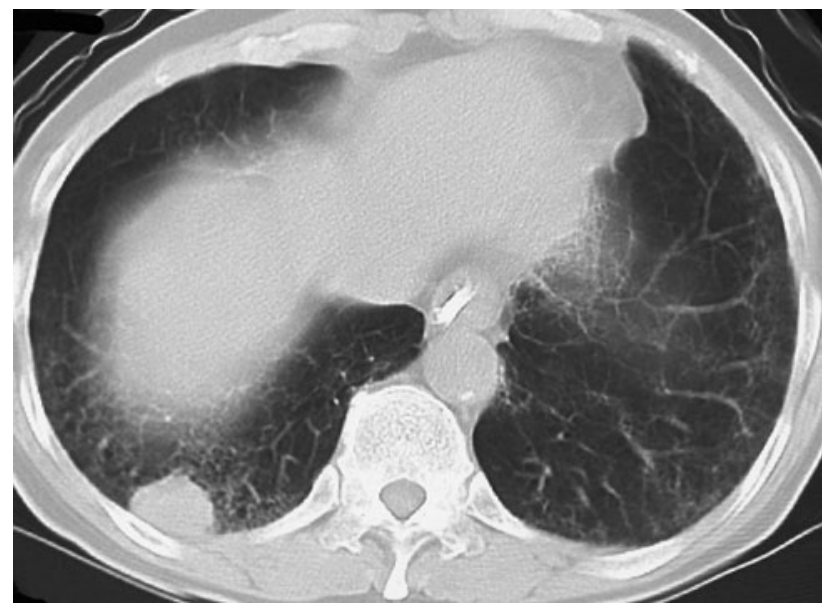

Fig. 1 CT findings on admission. A 2-cm-diameter mass in the bottom area of the lower lobe of the right lung

disturbance of the sensory system of his face, trunk, or limbs. His deep tendon reflexes were normal. Hematological and biochemical examinations were within the normal limits. Brain magnetic resonance imaging showed no abnormal lesions. At first, tetanus was suspected, although he had experienced no trauma. To maintain the airway, he was given a tracheostomy. Tetanus antitoxin, immune globulin, and the antibiotics penicillin $\mathrm{G}$ and clindamycin were administered, but his symptoms did not improve. The patient was given a gastrostomy for feeding.

Chest computed tomography (CT) showed a $2-\mathrm{cm}$ diameter mass in the bottom area of the lower lobe of the right lung (Fig. 1), a 3-mm-diameter small nodule of the middle lobe of the right lung, a 1-cm-diameter nodule of the lower lobe of the left lung, and lower paratracheal lymph node swelling of the mediastinum. Transbronchial biopsy was done from the right B9, and small cell carcinoma was diagnosed. The serum tumor markers carcinoembryonic antigen, pro-gastrin-releasing peptide, and CYFRA were within normal limits, but neuron-specific enolase was over the normal limit $(15.0 \mathrm{ng} / \mathrm{mL}$, normal range $0-12.0 \mathrm{ng} / \mathrm{mL}$ ). Paraneoplastic neurological syndrome was suspected, and so anti-Hu (ANNA-1), anti-Ri (ANNA-2), anti-Yo (PCA-1), anti-voltage-gated calcium channel, anti-voltage-gated potassium channel, anti-glutamic acid decarboxylase, anti-acetylcholine receptor (AChR), and anti-muscle-specific tyrosine kinase antibodies were checked; all were negative except for anti-AChR $(0.5 \mathrm{nmol} / \mathrm{L}$, normal range $<0.2 \mathrm{nmol} / \mathrm{L})$. A repetitive stimulation test of the nerves did not show waning or waxing, a tensilon test was negative, and no change in the symptoms was found within a single day. The motor nerve conduction velocities of median, ulnar, peroneal, and post-tibial nerves were normal, as were the sensory nerve conduction velocities of median, ulnar, and sural nerves.

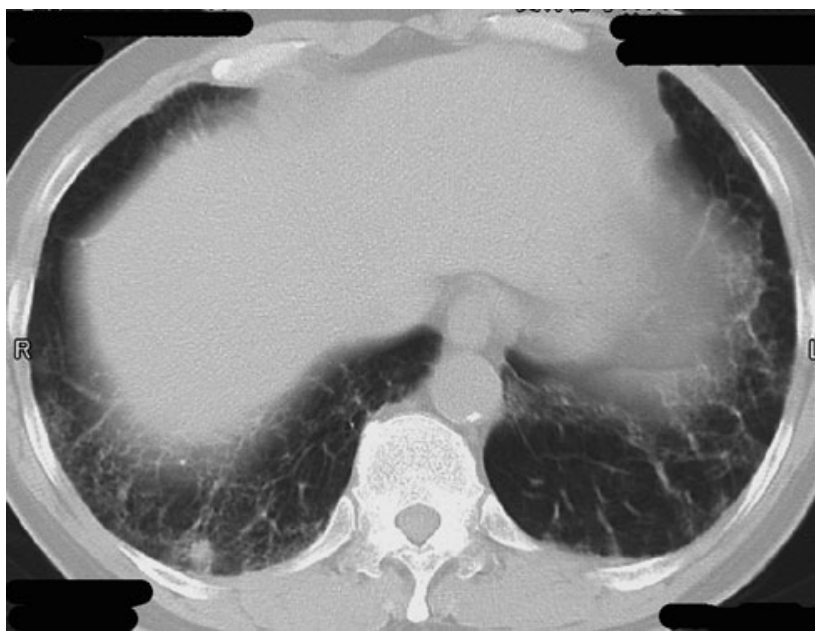

Fig. 2 CT findings after four cycles of chemotherapy for lung cancer. The primary mass of the right lung shrank, and the effect of chemotherapy reached a partial response

Upon the diagnosis of small cell lung cancer, cT2N2M1a (contralateral pulmonary metastasis) stage IV, four cycles of carboplatin and etoposide were administered. The patient's mouth-opening difficulty and diplopia began to improve after the first cycle, and both of these symptoms disappeared after three cycles. The artificial fistulae of the trachea for the airway and stomach for feeding were then closed. The effect of four cycles of chemotherapy was a partial response (Fig. 2). One month after the four cycles of the first chemotherapy were completed, his left arm began to itch, and the itch extended to the back of his neck, his right arm, the right side of his face, and both lower limbs. Four months after the four cycles of the first chemotherapy were completed, the primary mass of the right lung started to grow. Therefore, four cycles of carboplatin and etoposide were administered again, and the tumor shrank again. The itching improved after the first course of the secondline chemotherapy, and disappeared after the four cycles. One month after the four cycles of the second-line chemotherapy were completed, bilateral ptosis and itching appeared. Scars from scratching were found on both of his upper limbs. Cerebrospinal fluid (CSF) analysis revealed a protein level of $47 \mathrm{mg} / \mathrm{dL}$, three cells per three visual fields, and a sugar level of $75 \mathrm{mg} / \mathrm{dL}$. A CSF culture and serological analyses ruled out an infectious etiology. Compared with data in the first examination, the motor nerve conduction velocity was not changed, but the sensory nerve action potentials of the sensory nerve conduction velocities of the median, ulnar, and sural nerves were decreased. His symptoms were suspected to have been caused by paraneoplastic neurological syndrome, so immunoglobulin (400 mg/kg/day) was administered intravenously. The ptosis was improved but the itching was aggravated and dysphagia appeared 5 days after the 
Fig. 3 Clinical course. $P D$ progressive disease, $C B D C A$ carboplatin, $V P-16$ etoposide, $A M R$ amrubicin, $C D D P$ cisplatin, CPT-11 irinotecan, NSE neuron-specific enolase, IVIg intravenous immunoglobulin

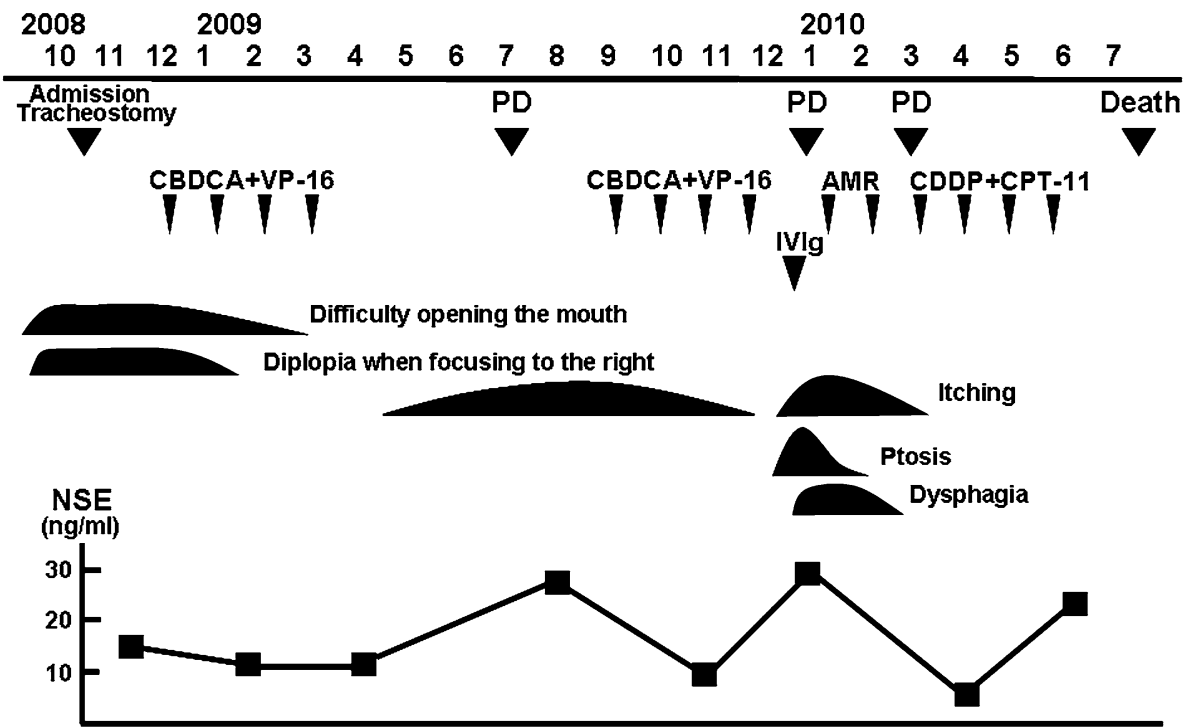

administration of immunoglobulin. A laryngeal fiberscope showed immortalization of the vocal cord. At that time, chest CT showed new pulmonary masses of the right lower lobe and swelling of the mediastinal and hilar lymph nodes. To treat the tumor relapse, two cycles of amrubicin were administered. The tumor and the lymph nodes shrank, and at the same time the symptoms improved. After 1 month of two cycles of the third-line chemotherapy, the tumor relapsed. Cisplatin and irinotecan were administered for four cycles, but this did not control the growth of the lung cancer lesions. Nineteen months after the start of the first chemotherapy for lung cancer, the patient died from obstructive pneumonia (Fig. 3).

\section{Discussion}

Difficulty in mouth opening can have a variety of causes, such as dental abscess, trauma, radiation of the facial muscles, and the side effects of medicines. It is very rare to discover cancer in the course of searching for the cause of difficulty in mouth opening. The Paraneoplastic Neurological Syndrome Euronetwork recommended a set of diagnostic criteria and management guidelines for paraneoplastic neurological syndromes [1-3]. They applied the classical syndrome to encephalomyelitis, limbic encephalitis, subacute cerebellar degeneration, opsoclonus-myoclonus, subacute sensory neuronopathy, chronic gastrointestinal pseudo-obstruction, Lambert-Eaton myasthenic syndrome, and dermatomyositis. Difficulty in mouth opening is a nonclassical syndrome of paraneoplastic neurological syndrome. However, treatment for small cell lung cancer clearly corrected this symptom, diplopia, and recurrent laryngeal nerve paralysis on first admission. In accordance with the diagnostic criteria of the Paraneoplastic Neurological Syndrome
Euronetwork, we diagnosed the patient's symptoms as paraneoplastic neurological syndrome [1]. We suspected that these symptoms were attributable to the disturbance of myoneural junctions. We did not find characteristic onconeural antibodies but rather acetylcholine receptor antibody, which is related to myasthenia gravis of thymoma. The patient did not show thymoma, weaning upon repetitive stimulation tests of the nerves, reactivity to a tensilon test, or any change of the symptoms within a single day. This indicated that the patient did not have myasthenia gravis. In this patient, tumor relapse was accompanied by bilateral ptosis, itching, and dysphagia. Chronic itching is a component of paraneoplastic syndrome, which occurs in hematological malignancies with skin lesions [4]. In the present case, skin lesions presented as a result of the itch-scratch cycle. The itching, ptosis, and dysphagia were also clearly eliminated by chemotherapy for lung cancer, suggesting the occurrence of paraneoplastic syndrome. Intravenous immunoglobulin administration did not improve the itching or dysphagia. In an examination conducted after the tumor relapse, the sensory nerve action potential of the sensory nerve conduction velocity was decreased in comparison to that in the first examination. This might suggest that peripheral nerves were involved in this syndrome. Because paraneoplastic syndromes are considered immune-mediated, removal of the antigen by treatment of the tumor is expected to improve the symptoms. We suspected that the variation of symptoms in this case was attributable to the change of antigens accompanied by chemotherapy and tumor relapse. Darnell and Posner [5] proposed that the apoptosis of tumor cells triggers an antitumor immune response, in which apoptotic cells are phagocyted by dendric cells that migrate to lymph nodes, where they activate antigen-specific $\mathrm{CD} 4+, \mathrm{CD} 8+$, and $\mathrm{B}$ cells, and the $\mathrm{B}$ cells mature into plasma cells that produce antibodies against the tumor antigens. These mechanisms 
might induce variation of tumor antigens. Mouth-opening difficulty and itching are very rare symptoms of small cell lung cancer. This case showed that difficulty in mouth opening could be a paraneoplastic neurological symptom that occurs before cancer is diagnosed. The origins of these symptoms might be attributable to a new etiology.

Conflict of interest Authors declare that they have no conflict of interest

\section{References}

1. Graus F, Delattre JY, Antoine JC et al (2004) Recommended diagnostic criteria for paraneoplastic neurological syndromes. J Neurol Neurosurg Psychiatry 75:1135-1140
2. Vedeler CA, Antoine JC, Giometto B et al (2006) Paraneoplastic Neurological Syndrome Euronetwork. Management of paraneoplastic neurological syndromes: report of an EFNS Task Force. Eur J Neurol 13:682-690

3. Titulaer MJ, Soffietti R, Dalmau J et al (2011) Screening for tumours in paraneoplastic syndromes: report of an EFNS task force. Eur J Neurol 18:19-27

4. Yosipovitch G (2010) Chronic pruritus: a paraneoplastic sign. Dermatol Ther 23:590-596

5. Darnell RB, Posner JB (2003) Paraneoplastic syndromes involving the nervous system. N Engl J Med 349:1543-1554 